Forum 2018 · 33:219

https://doi.org/10.1007/s12312-018-0437-9

Online publiziert: 20. Juni 2018

(c) Springer Medizin Verlag GmbH, ein Teil von

Springer Nature 2018

\title{
Leitlinienprogramm Onkologie legt S3-Leitlinie zum Endometriumkarzinom vor
}

mendem Lebensalter. Erste Anzeichen sind oft ungewöhnliche Blutungen aus der Scheide. 80 \% der Endometriumkarzinome werden im Stadium FIGO I diagnostiziert. In diesem Stadium lassen sich durch eine Operation und gegebenenfalls eine Bestrahlung Überlebensraten zwischen 81 und $90 \%$ erzielen.

Beim operativen Eingriff entfernt der Operateur die gesamte Gebärmutter sowie die Eierstöcke und Eileiter beidseits. Abhängig vom Stadium kann außerdem die Entfernung angrenzender Gewebe und der Lymphknoten im kleinen Becken nötig sein - der Lymphknotenbefall ist ein wichtiger prognostischer Marker. Aufgrund der Studienlage kommen die Autoren der Leitlinie allerdings zum Schluss, dass eine systematische Entfernung dieser Lymphknoten bei Patientinnen mit einem östrogenabhängigen Typ-I-Karzinom im Frühstadium (pT1a, G1/2) nicht als Routineverfahren empfohlen werden kann. Die aktuelle Studienlage spricht auch gegen eine adjuvante Chemo- oder Strahlentherapie bei frühen Typ-I-Endometriumkarzinomen im Stadium pTla/b G1 und G2 cN0/pN0.

Die Bedürfnisse der Patientinnen nach Diagnose und Therapie eines Endometriumkarzinoms sind in der Regel sehr komplex. Als Folgen der Erkrankung bzw. der
Therapie können Bauchdecken- und Adhäsionsbeschwerden, sexuelle Funktionsstörungen, Schmerzen beim Geschlechtsverkehr, Scheidentrockenheit sowie Harnblasen- und Darmstörungen auftreten. „Solche Beschwerden müssen nicht nur in der Primärtherapie, sondern auch im Rahmen der Rehabilitation und in der Nachsorge erfragt und behandelt werden", sagt Professor Emons. Es sei wichtig, dass die Behandlung durch kompetente und interdisziplinäre Teams durchgeführt werde und dass die Patientinnen eingehend über die gesetzlichen Möglichkeiten zur Beantragung und Inanspruchnahme von Rehabilitationsleistungen informiert und beraten werden.

Für den direkten Zugang zur neuen S3Leitlinie nutzen Sie bitte folgenden Link: www.leitlinienprogramm-onkologie.de/ leitlinien/endometriumkarzinom/

\section{Korrespondenzadresse}

\section{Dr. Katrin Mugele}

Pressestelle der Deutschen Krebsgesellschaft e.V. mugele@krebsgesellschaft.de

\section{Luise Dusatko}

Pressestelle der Deutschen Gesellschaft für Gynäkologie und Geburtshilfe e.V. presse@dggg.de kungsalter liegt aktuell bei etwa 69 Jahren, die Neuerkrankungsrate steigt mit zuneh- 